\title{
Phenotypic detection of metallo- $\beta$-lactamases in Pseudomonas aeruginosa and Acinetobacter baumannii isolated from hospitalized patients in São Luis, State of Maranhão, Brazil
}

\author{
Roberto Morais Luz de Carvalho[1], Sirlei Garcia Marques ${ }^{[2]}$, Luís Henrique Bastos Gonçalves ${ }^{[3]}$, \\ Afonso Gomes Abreu ${ }^{[1]}$, Silvio Gomes Monteiro ${ }^{[3]}$ and Azizedite Guedes Gonçalves ${ }^{[1],[4]}$
}

[1]. Programa de Pós-Graduação em Ciências da Saúde, Universidade Federal do Maranhão, São Luis, MA. [2]. Hospital Universitário Unidade Presidente Dutra, Universidade Federal do Maranhão, Presidente Dutra, MA. [3]. Departamento de Pós-Graduação, Centro Universitário do Maranhão, São Luis, MA. [4]. Departamento de Patologia, Universidade Federal do Maranhão, São Luis, MA.

\begin{abstract}
Introduction: Acquired metallo- $\beta$-lactamases $(\mathrm{M} \beta \mathrm{L})$ are emerging determinants of resistance in Pseudomonas aeruginosa and Acinetobacter baumannii. The objectives of this study were to phenotypically detect $\mathrm{M} \beta \mathrm{L}$ in imipenem-resistant $P$. aeruginosa and A. baumannii, to investigate the association between MBL-positive strains and hospitals, and to compare the resistance profiles of M $\beta \mathrm{L}$-producing and non-M $\beta \mathrm{L}$-producing strains. Methods: The approximation disk and combined disk assay methods were used in this study. Results: A total of 18 (38.3\%) P. aeruginosa isolates and 1 (5.6\%) A. baumannii isolate tested positive for the presence of M $\beta \mathrm{L}$. Conclusions: These results demonstrate the need for strict surveillance and for the adoption of preventive measures to reduce the spread of infection and potential outbreaks of disease caused by M $\beta \mathrm{L}$-producing microorganisms.
\end{abstract}

Keywords: Metallo- $\beta$-lactamases. Pseudomonas aeruginosa. Acinetobacter baumannii.

Metallo- $\beta$-lactamase $(\mathrm{M} \beta \mathrm{L})$ activity has emerged as one of the most feared resistance mechanisms because of the ability of M $\beta$ Ls to hydrolyze virtually all $\beta$-lactam agents, including carbapenems. However, M $\beta$ Ls are unable to hydrolyze monobactams because their genes are carried on highly mobile elements. The prevalence of metallo- $\beta$-lactamase-producing Pseudomonas aeruginosa (MPPa) causing nosocomial infections has been increasing worldwide ${ }^{1}$.

Data from the SENTRY Antimicrobial Surveillance Program suggest $44.8 \%$ of $P$. aeruginosa isolates in Brazil are resistant to imipenem, and $43.9 \%$ of these isolates produce $\mathrm{M} \beta \mathrm{L}^{2}$.

The objectives of the present study were to phenotypically detect M $\beta \mathrm{L}$ in imipenem-resistant Pseudomonas aeruginos $a$ and Acinetobacter baumannii isolates, to investigate the association between M $\beta \mathrm{L}$-positive strains and the hospitals studied, and to compare the resistance profiles of $\mathrm{M} \beta \mathrm{L}$-producing and nonM $\beta$ L-producing strains.

A total of 129 consecutive $P$. aeruginosa and 71 A. baumannii isolates were recovered between June and November 2008. The strains were isolated from different clinical samples obtained from 2 private hospitals (Hospital 1: 100 beds and Hospital 3: 164 beds) and 1 public hospital (Hospital 2: 121 beds) in

Address to: Dra Azizedite Guedes Gonçalves. Depto ${ }^{\circ}$. Patologia/UFMA. Rua Madre Deus 02, 65025-560 São Luis, MA, Brasil.

Phone: 5598 3235-0170

e-mail: azizeg@ig.com.br

Received 5 November 2011

Accepted 18 May 2012
São Luis, State of Maranhão, northeastern Brazil. All isolates were identified both by conventional techniques and by the automated Vitek 2 system (BioMérieux ${ }^{\circledR}$, Marcy l'Etoile, France). Among those isolates, 47 (36.4\%) P. aeruginosa isolates and $18(25.4 \%)$ A. baumannii isolates were resistant to imipenem as determined by the Clinical and Laboratory Standards Institute (CLSI) disk diffusion method (inhibition zone of $\leq 13 \mathrm{~mm}$ or $\mathrm{MIC} \geq 16 \mu \mathrm{g} / \mathrm{mL}$ ); these isolates were characterized.

The disk diffusion method (Kirby-Bauer) was used for antimicrobial susceptibility testing according to the recommendations of the CLSI found in performance standard M100-S22 (2012). Quality control testing was performed using P. aeruginosa ATCC 27853 and Escherichia coli ATCC 25922.

The disk approximation test was performed according to the method of Arakawa et al. ${ }^{3}$, with modifications to the inhibitor volumes and concentrations. For the detection of M $\beta \mathrm{L}$ production by $P$. aeruginosa, 2 ceftazidime disks $(30 \mu \mathrm{g}$; Oxoid $^{\circledR}$, Basingstoke, England) were used as the substrates and were placed at center-to-center distances of 2.0 and $1.5 \mathrm{~cm}$ from 2 un-impregnated disks. Next, $4 \mu \mathrm{L}$ of 2-mercaptopropionic acid (2-MPA, 1.4mM; Sigma-Aldrich ${ }^{\circledR}$, Steinheim, Germany) was added to the first disk, and $4 \mu \mathrm{L}$ of ethylenediaminetetraacetic acid (EDTA, 400mM; Sigma-Aldrich ${ }^{\circledR}$, Steinheim, Germany) was added to the second disk. This assay was repeated for A. baumannii using imipenem $(10 \mu \mathrm{g})$ as the substrate and $4 \mu \mathrm{L}$ of 2-MPA $(1.4 \mathrm{mM})$ and $8 \mu \mathrm{L}$ of EDTA $(200 \mathrm{mM})$ as inhibitors. The plates were incubated for $18-24 \mathrm{~h}$ at $35^{\circ} \mathrm{C}$.

For the combined disk assay, the inhibitor concentrations, pipetted volumes, and cut-off values for the differentiation of producers and non-producers of $\mathrm{M} \beta \mathrm{L}$ were determined 
according to Picão et al. ${ }^{4}$. Three ceftazidime (CAZ) disks were placed on the surface of Mueller-Hinton plates that were inoculated with a suspension of $P$. aeruginosa. Next, $8 \mu \mathrm{L}$ of 2-MPA $(1.4 \mathrm{mM})$ was added to the first disk, $8 \mu \mathrm{L}$ of EDTA $(100 \mathrm{mM})$ was added to the second disk, and no inhibitor was added to the third disk to determine the diameter of the halo formed in the absence of an inhibitor. This assay was repeated for $A$. baumannii using imipenem $+2-\mathrm{MPA}(4 \mu \mathrm{L}, 1.4 \mathrm{mM})$ and imipenem+EDTA $(4 \mu \mathrm{L}, 400 \mathrm{mM})$. The plates were incubated for $18-24 \mathrm{~h}$ at $35^{\circ} \mathrm{C}$. The diameter of the inhibition halo produced by the $\beta$-lactam disks that contained the inhibitors was compared to the halo diameter produced by the antibiotic-only disk. The result was defined as positive when, compared to the antibiotic-only disk, the increase in the diameters produced by the $\mathrm{EDTA}+\mathrm{CAZ}$ or 2-MPA+CAZ disks was greater than 8 or $14 \mathrm{~mm}$, respectively. For $A$. baumannii, $\mathrm{M} \beta \mathrm{L}$ production was defined as an increase in the diameter of the inhibition halo of $>4 \mathrm{~mm}$ for imipenem+2-MPA and $>$ for imipenem+EDTA compared to the imipenem-only disk.

Pseudomonas aeruginosa ATCC 27853 was used as the negative control. P. aeruginosa (PSA319) and A. baumannii strains provided by the Laboratory of Clinical Microbiology, Universidade Federal de São Paulo (LEMC), and the University Hospital of Universidade Federal de Santa Catarina, respectively, were used as positive controls for $\mathrm{M} \beta \mathrm{L}$ production.

The results were analyzed using the Statistical Package for the Social Sciences (SPSS) for Windows, version 20.0 (2012). The chi-squared test for independent samples was applied to evaluate the association of MPPa or non-M $\beta \mathrm{L}$-producing $P$. aeruginosa (NPPa) strains with each hospital and with antibiotic resistance. A level of significance of $5 \%(\mathrm{p}<0.05)$ was adopted for all tests.

Strains of $P$. aeruginosa resistant to imipenem were recovered from $68(52.7 \%)$ tracheal secretion samples, $18(14 \%)$ urine samples, $10(7.8 \%)$ catheter tip samples, 9 (7\%) blood samples, 7 (5.4\%) samples from sores/wounds, and 17 (13.1\%) other biological samples. For isolates of $A$. baumannii that were resistant to imipenem, the most frequent site of strain isolation was tracheal secretion, $40(56.3 \%)$; followed by the catheter tip, 12 (16.9\%); blood, 5 (7\%); urine, 5 (7\%); and other biological samples, $9(12.8 \%)$.
The imipenem resistance profiles of $P$. aeruginosa and A. baumannii did not differ significantly among the hospitals studied (Table 1), and the phenotypic detection of $\mathrm{M} \beta \mathrm{L}$ was positive in $18(38.3 \%)$ P. aeruginosa isolates and 1 (5.6\%) A. baumannii isolate. The results from the 2 methods used were in agreement.

A total of $12(66.7 \%) \mathrm{MPPa}$ isolates were collected from Hospital 1, and $6(33.3 \%)$ were collected from Hospital 3. Furthermore, there was a significant difference in the proportion of MPPa and NPPa among the 3 hospitals ( $\mathrm{p}=0.0016$ ).

The highest percentage of MPPa isolation was derived from catheter tips, 6 (33.3\%); followed by urine, 5 (27.8\%); tracheal secretions, 3 (16.7\%); bronchoalveolar lavage fluid, 1 (5.6\%); peritoneal fluids, 1 (5.6\%); blood, 1 (5.6\%); and nose secretions, 1 (5.6\%).

In total, $62.1 \%, 48.3$ and $89.7 \%$ of the NPPa isolates were resistant to cefepime, ceftazidime, and meropenem, respectively; all MPPa isolates were resistant to these three compounds. The resistance rate was higher among MPPa isolates, with significant differences for amikacin $(\mathrm{p}=0.0264)$, cefepime ( $\mathrm{p}=0.0085)$, ceftazidime $(\mathrm{p}=0.0007)$, and piperacillin/ tazobactam $(\mathrm{p}=0.0119)$. Aztreonam resistance was higher in the NPPa group $(\mathrm{p}=0.0196)$. None of the $P$. aeruginosa isolates were resistant to polymyxin B (Table 2).

The only strain of M $\beta$ L-producing A. baumannii (MPAb) was isolated from the blood sample of an Intensive Care Unit patient (ICU) from Hospital 1. This strain was resistant to amikacin, gentamicin, cefepime, ceftazidime, imipenem, meropenem, and ciprofloxacin and was susceptible to ampicillin/ sulbactam and piperacillin/tazobactam.

Multidrug-resistant $P$. aeruginosa and Acinetobacter strains are becoming a worldwide problem. SENTRY data have revealed that the prevalence of antimicrobial resistance among P. aeruginosa isolates has been increasing in Latin American medical centers ${ }^{2}$. The arrival of $\mathrm{M} \beta \mathrm{L}$ emphasizes the importance of phenotypic investigations for the presence of $\mathrm{M} \beta \mathrm{L}$ in routine laboratory tests 5 .

Monitoring programs in Brazil have indicated that the prevalence of $P$. aeruginosa isolates that are resistant to

TABLE 1 - Imipenem resistance profiles of Pseudomonas aeruginosa and Acinetobacter baumannii strains isolated between June and November 2008, from 3 hospitals in São Luis, State of Maranhão, Brazil.

\begin{tabular}{|c|c|c|c|c|c|c|c|c|c|c|c|c|c|c|c|c|}
\hline & \multicolumn{8}{|c|}{ Pseudomonas aeruginosa } & \multicolumn{8}{|c|}{ Acinetobacter baumannii } \\
\hline & $\mathrm{n}$ & $\%$ & $\mathrm{n}$ & $\%$ & $\mathrm{n}$ & $\%$ & $\mathrm{n}$ & $\%$ & $\mathrm{n}$ & $\%$ & $\mathrm{n}$ & $\%$ & $\mathrm{n}$ & $\%$ & $\mathrm{n}$ & $\%$ \\
\hline Hospital 1 & 19 & 35.2 & 6 & 11.1 & 29 & 53.7 & 54 & 100.0 & 8 & 30.8 & 0 & 0.0 & 18 & 69.2 & 26 & 100.0 \\
\hline Hospital 2 & 19 & 38.8 & 0 & 0.0 & 30 & 61.2 & 49 & 100.0 & 1 & 9.1 & 0 & 0.0 & 10 & 90.9 & 11 & 100.0 \\
\hline Total & 47 & 36.4 & 6 & 4.7 & 76 & 58.9 & 129 & 100.0 & 18 & 25.4 & 0 & 0.0 & 53 & 74.6 & 71 & 100.0 \\
\hline$\chi^{2}$ & \multicolumn{6}{|c|}{8.93} & \multicolumn{10}{|c|}{1.96} \\
\hline $\mathrm{P}$ & \multicolumn{6}{|c|}{0.0628} & \multicolumn{10}{|c|}{0.3748} \\
\hline
\end{tabular}


TABLE 2 - The chi-squared test for independent samples comparing the antibiotic resistance profiles of M $\beta \mathrm{L}$-producing and non-M $\beta \mathrm{L}$-producing Pseudomonas aeruginosa strains isolated between June and November 2008 from 3 hospitals in São Luis, State of Maranhão, Brazil.

\begin{tabular}{lcccc}
\hline Antibiotic & MPPa $(\%)$ & $\mathrm{NPPa}(\%)$ & $\chi^{2}$ & $\mathrm{p}$ \\
\hline Amikacin $(30 \mu \mathrm{g})$ & 77.8 & 44.8 & 4.93 & 0.0264 \\
Aztreonam $(30 \mu \mathrm{g})$ & 5.6 & 41.4 & 5.44 & 0.0196 \\
Cefepime $(30 \mu \mathrm{g})$ & 100.0 & 62.1 & 6.92 & 0.0085 \\
Ceftazidime $(30 \mu \mathrm{g})$ & 100.0 & 48.3 & 11.39 & 0.0007 \\
Ciprofloxacin $(5 \mu \mathrm{g})$ & 94.4 & 75.9 & 2.71 & 0.0994 \\
Gentamicin $(10 \mu \mathrm{g})$ & 88.9 & 72.4 & 1.80 & 0.1797 \\
Meropenem $(10 \mu \mathrm{g})$ & 100.0 & 89.7 & 0.63 & 0.4257 \\
Piperacillin/tazobactam $(100 / 10 \mu \mathrm{g})$ & 72.2 & 34.5 & 6.33 & 0.0119 \\
\hline
\end{tabular}

MPPa: metallo- $\beta$-lactamase-producing Pseudomonas aeruginosa; NPPa: non-metallo- $\beta$ lactamase-producing Pseudomonas aeruginosa.

carbapenems is between $12 \%$ and $19 \%$, and $6 \%$ to $9 \%$ of the Acinetobacter spp. isolates are also resistant ${ }^{6}$. The proportions of MPPa and MPAb have been reported in a few studies in Brazil, but all such studies were restricted to the southeastern or southern regions of the country ${ }^{5,7-10}$.

Epidemiological studies tracking the prevalence of M $\beta \mathrm{L}-$ producing microorganisms in northeastern Brazil are scarce ${ }^{11,12}$; therefore, it is necessary to adopt preventive measures and to establish effective treatment protocols that are appropriate for each hospital and region.

Machado et al..$^{5}$ detected 17.4\% MPPa and 6.3\% MPAb in a hospital in the State of Rio Grande do Sul, while Graf et al. ${ }^{8}$ detected 36\% MPPa in that the same state. In Hospital São Paulo, State of São Paulo, Sader et al. ${ }^{9}$ found a prevalence of $19.7 \%$ MPPa in blood samples. Cipriano et al. ${ }^{13}$ detected the presence of the São Paulo Metallo- $\beta$-lactamase gene (SPM) in strains of $P$. aeruginosa from the same city examined in the current study. Regarding Acinetobacter spp., Tognin et al. ${ }^{14}$ found that the prevalence of M $\beta \mathrm{L}$ producers in São Paulo Hospital rose from $0 \%$ between 1993 and 1997 to $29 \%$ in 1998 and finally to $100 \%$ between 1999 and 2001. In our study, the proportion of MPPa was $38.3 \%$, which is high compared to other states. For A. baumannii, we determined the rate of $\mathrm{M} \beta \mathrm{L}$ producers was $5.6 \%$, which is similar to the value reported by Machado et al. ${ }^{5}$.

The higher prevalence of resistance to cefepime, ceftazidime, ciprofloxacin, meropenem, amikacin, gentamicin, and piperacillin/ tazobactam observed in the MPPa group is consistent with the results of Machado et al. ${ }^{5}$ and Zavascki et al. ${ }^{7}$; however, resistance in the NPPa group for our study was higher than the values reported by these authors. Normally, P. aeruginosa isolates are resistant to $\beta$-lactam antibiotics because of the hyperproduction of AmpC $\beta$-lactamase. In addition, the presence of an efflux pump and the alteration of the outer membrane permeability may play important roles by restricting the access of antimicrobial agents to their intracellular targets. These resistance mechanisms developed by $P$. aeruginos $a$ may explain the high prevalence of resistance among non-M $\beta \mathrm{L}$-producing strains that was observed in this study. The resistance of MPPa to aminoglycosides was most likely due to the spread of other resistance determinants that occur concomitantly with the dissemination of $\mathrm{M} \beta \mathrm{L}$, such as the expression of genes that encode aminoglycoside-modifying enzymes in class 1 integrons. These factors explain, in part, the cases of multidrug resistance ${ }^{1}$. There was a statistically significant difference in the proportion of resistance to the antibiotics amikacin, aztreonam, cefepime, ceftazidime, and piperacillin/ tazobactam between the MPPa and NPPa groups (Table 2).

Although the literature states that $\mathrm{M} \beta \mathrm{L}$-producing microorganisms hydrolyze all commercially available ß-lactams except aztreonam, we identified 1 isolate that was completely resistant and 3 isolates that were partially resistant to this antibiotic. In addition, all isolates tested positive for M $\beta \mathrm{L}$. The isolate identified here may be the same clone as reported by Cipriano et al..$^{13}$ because the present study was conducted in the same city (although during a different period of time).

The MBL-producing A. baumannii isolate detected in our study exhibited resistance to most antimicrobials, limiting the therapeutic options to ampicillin-sulbactam and piperacillintazobactam among the antibiotics tested. The emergence of carbapenem resistance has limited the therapeutic options for A. baumannii infections to polymyxins and ampicillin/sulbactam ${ }^{14}$. MPPa and MPAb are isolated with increasing frequency in Brazil and worldwide; therefore, understanding the prevalence and resistance of these strains has become critically important. These microorganisms are the etiological agents of most ICU-acquired infections, particularly infections of the respiratory tract; these infections are typically severe, and there are limited therapeutic options to treat them. These infections are directly responsible for the high rates of morbidity and mortality and the prolongation of hospital stays with concomitant high hospital costs. This scenario demonstrates the need for strict surveillance by infection control committees in the health care community and for the adoption of preventive measures to reduce the spread of infection and potential outbreaks caused by M $\beta \mathrm{L}$-producing microorganisms.

\section{ACKNOWLEDGMENTS}

We thank the Cedro Laboratory for kindly providing the physical space and materials used in this study, Dr. Nilton Lincopan and Dr. Líbera Maria Dalla Costa for donating essential reagents, and the technician Rosália de Fátima Martins for helping with the tests and data organization.

\section{CONFLICT OF INTEREST}

The authors declare that there is no conflict of interest.

\section{REFERENCES}

1. Walsh TR. Emerging carbapenemases: a global perspective. Int Journal Antimicrob Agents 2010; 36 (supl III):8-14.

2. Fritsche TR, Sader HS, Toleman MA, Walsh TR, Jones RN. Emerging metallo- $\beta$-lactamase-mediated resistances: A summary report from the worldwide SENTRY antimicrobial surveillance program. Clin Infect Dis 2005; 41:276-278. 
3. Arakawa Y, Shibata N, Shibayama K, Kurokawa H, Yagi T, Fujiwara $\mathrm{H}$, et al. Convenient test for screening metallo- $\beta$-lactamase-producing gram-negative bacteria by using thiol compounds. J Clin Microbiol 2000; 38:40-43.

4. Picão RC, Andrade SS, Nicoletti AG, Campana EH, Moraes GC, Mendes $\mathrm{RE}$, et al. Metallo- $\beta$-lactamase detection: comparative evaluation of doubledisk synergy versus combined disk tests for IMP-, GIM-, SIM-, SPM-, or VIM-producing isolates. J Clin Microbiol 2008; 46:2028-2037.

5. Machado GM, Lago A, Fuentefria SRR, Fuentefria DB. Ocurrence and the susceptibility to antimicrobial agents in Pseudomonas aeruginosa and Acinetobacter sp. at a tertiary hospital in southern Brazil. Rev Soc Bras Med Trop 2011; 44:168-172.

6. Furtado GHC, Martins ST, Machado AMO, Wey SB, Medeiros EAS. Clinical culture surveillance of carbapenem-resistant Pseudomonas aeruginosa and Acinetobacter species in a teaching hospital in São Paulo, Brazil: A 7-year study. Infect Control Hosp Epidemiol 2006; 27:1270-1273.

7. Zavaski AP, Barth AL, Gaspareto PB, Gonçalves ALS, Moro ALD, Fernandes $\mathrm{JF}$, et al. Risk factors for nosocomial infections due to Pseudomonas aeruginosa producing metallo- $\beta$-lactamase in two tertiary-care teaching hospitals. J Antimicrob Chemother 2006; 58:882-885.

8. Gräf T, Fuentefria DB, Corção G. Occurrence of multiresistant strains of Pseudomonas aeruginosa producing metallo-b-lactamase bla clinical samples. Rev Soc Bras Med Trop 2008; 41:306-308.
9. Sader HS, Reis AO, Silbert S, Gales AC. IMPs, VIMs, and SPMs: the diversity of metallo- $\beta$-lactamases produced by carbapenem-resistant Pseudomonas aeruginosa in a Brazilian hospital. Clin Microbiol Infect 2004; 11:73-76.

10. Laranjeira VS, Marchetti DP, Steyer JR, Corção G, Picoli SU. Pesquisa de Acinetobacter sp e Pseudomonas aeruginosa produtores de metalo$\beta$-lactamase em hospital de emergência de Porto Alegre, Estado do Rio Grande do Sul, Brasil. Rev Soc Bras Med Trop 2010; 43:462-464.

11. Santos Filho L, Santos IB, Assi AML, Xavier DL. Determinação da Produção de metalo beta-lactamases em amostras de Pseudomonas aeruginosa isoladas em João Pessoa, Paraíba. J Bras Patol Med Lab 2002; 38:291-296.

12. Torres JCN, Menezes EA, Ângelo MRF, Oliveira IRN, Salviano MNC, Xavier DE, et al. Cepas de Pseudomonas spp. produtoras de metalobetalactamase isoladas no Hospital Geral de Fortaleza. J Bras Patol Med Lab 2006; 42:313-319.

13. Cipriano R, Vieira VV, Fonseca EL, Rangel K, Freitas FS, Vicente AC. Coexistence of epidemic colistin-only sensitive clones of Pseudomonas aeruginosa, including the blaSPM clone, spread in hospital in a Brazilian Amazon City. Microb Drug Resist 2007; 13:142-146.

14. Tognim MCB, Gales AC, Penteado AP, Silbert S, Sader HS. Dissemination of IMP-1 metallo- $\beta$-lactamase-producing Acinetobacter species in a Brazilian teaching hospital. Infect Control Hosp Epidemiol 2006; 27:742-747. 